\title{
Urgences
}

\section{Voyager dans le temps des images suivi de Espace Laminé. Coupes à partir de sculptures}

\section{Marie-Chrystine Landry et Lise Lamarche}

Numéro 30, décembre 1990

L'autre du texte

URI : https://id.erudit.org/iderudit/025628ar

DOI : https://doi.org/10.7202/025628ar

Aller au sommaire du numéro

Éditeur(s)

Urgences

ISSN

0226-9554 (imprimé)

1927-3924 (numérique)

Découvrir la revue

Citer cet article

Landry, M.-C. \& Lamarche, L. (1990). Voyager dans le temps des images suivi de Espace Laminé. Coupes à partir de sculptures. Urgences, (30), 79-90.

https://doi.org/10.7202/025628ar d'utilisation que vous pouvez consulter en ligne. 


\title{
Voyager dans le temps des images Marie-Chrystine Landry
}

\author{
suivi de
}

Espace laminé.

Coupes à partir de sculptures.

Lise Lamarche 
Les premières oeuvres de ma démarche (axée sur l'architecture comme frange d'identité culturelle) ont été construites avec du bois récupéré provenant de caisses de kiwis, de raisins et d'oranges. Ce bois, de qualité emballage, souvent exotique par sa provenance, porte des marques à chaud aux noms inaltérables et lointains: Australie, Nouvelle-Zélande, Brésil, Italie, Maroc.

Des caisses donc, ou peut-être des caissettes, mais surtout de petites constructions aux formes simples, utiles, identifiées, auxquelles viendront se superposer des images à qui ma mémoire géo-ethnographique servira d'odomètre pour calculer leur parcours, prendre la mesure de leur route jusqu'à mon atelier.

Ce qui restera de leur provenance, ce sont des mots et des images (favelas, cathédrales, minarets et bungalows), ce sont aussi d'autres contenus. Ce qui restera de leur voyage en bateau me fera distinguer une rive: celle d'où je viens. Du fond d'une cuisine (à Saint-Henri, en 1987), je démembrais, planche par planche, toutes ces petites utilités en prenant garde de bien faire payer le billet aux minuscules passagères clandestines. Par la fenêtre, je voyais l'arrière d'un trois étages avec balcon amarré, comme un navire, à de précaires boîtes grises, en escale. Appuyée contre une balustrade, je surveillais un débarquement imaginaire.

C'est ainsi que mon travail se voudra d'ici et d'ailleurs, quotidien, d'un matériel simple et abondant.

\section{Là où l'itinéraire se complique}

Entre l'ici et l'ailleurs, je construirai des intervalles.

La reconstruction commence. Les petits tas de planches s'alignent sur le rebord de l'établi. Je peux tenir le contenu d'un lourd camion de madriers dans ma main. Jusque-là, c'est léger et c'est amusant de dominer tout ce matériel.

Armée d'un fusil à colle chaude (celui qui cimente au lieu de disperser), j'élabore les premières constructions. Elles s'imprègnent de l'Italie, des abords d'un fleuve vénézuélien, d'un roman de Duras où un barrage ne retient rien. Les constructions se multiplient, s'étirent pour me rejoindre, grimpent sur des échasses, perdent pied, quittent le ferme et se renforcent de partout pour s'assurer, pour moins vaciller dans le 


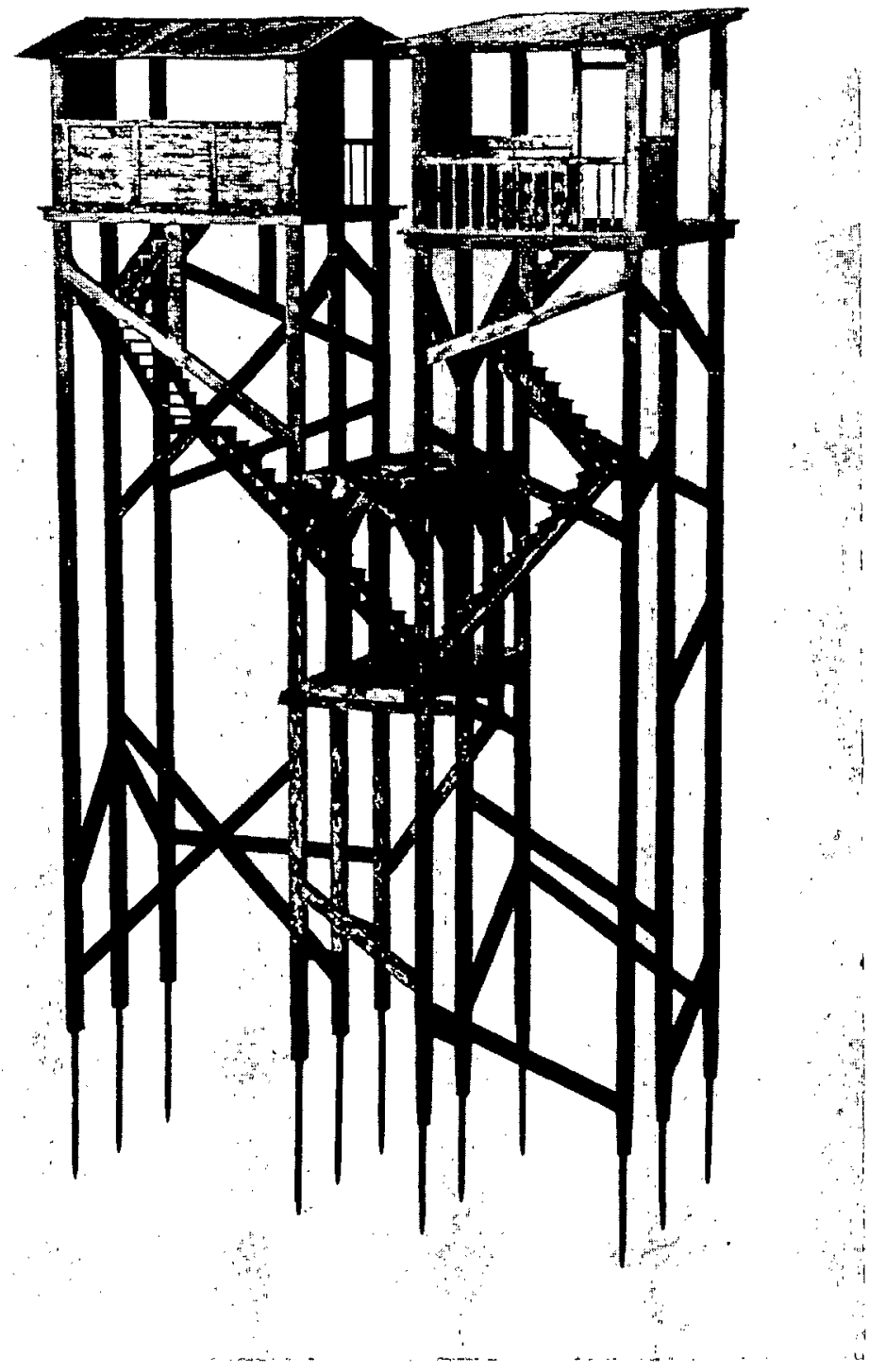

Marie-Chrystine Landry, Attente - bois, tôle, stuc, marbre $96 \mathrm{~cm} \times 52 \mathrm{~cm} \times 25 \mathrm{~cm}, 1988$ 
82

réel. Tout à coup, mes sculptures me regardent, me confrontent. Face à face, nous occupons le même nombre de centimètres cubes, la même quantité d'espace. Plantées là, nous portons le même regard ouvert, un peu ébahies, un peu dévastées, sur ce qui nous entoure. Je deviens objet comme mes objets, je deviens objet de mes objets qui comprennent vite qu'il n'y a de place pour les autres que dans le point de vue. Il importe peu, maintenant, de faire grand. L'échèlle et la problématique de l'espace dans l'objet s'imposent, la situation de l'objet dans l'espace devient aléatoire au gré des déplacements. Il n'est pas difficile de bouger quand la fascination s'exerce ailleurs.

\section{Là où le temps s'impose}

La construction, oui, c'est long, malgré le fait que j'aie pu croire un moment qu'en faisant plus petit, l'abattage et l'avancement de la démarche se feraient plus rapidement. Eh bien non! Je me barre les pieds dans des signes, je bourre mes coins de murs d'intentions d'atmosphère, j'invente des fonctions à des lieux et vice-versa. Je change de métier en cours de route, je deviens coloriste et peins des motifs d'un bord à l'autre. En cours de voûte, je deviens murator: les couleurs seront rabattues, usées, patinées par le temps, les textures et la matière garderont les marques de la scie et de la truelle qui mordent. Pas de temps à perdre avec la politesse du papier de verre.

Je détourne et conserve des matériaux, des traces, des parties d'éléments voués à la décrépitude temporelle en les retirant de leur trajectoire et en les réactualisant dans un autre ordre (celui de mon imaginaire) et dans un autre temps (celui des oeuvres). Je prélève de minces tranches de bois sur des éléments architecturaux réels pour en conserver les marques et couleurs altérées par le temps. Je découpe la forme pour en garder une part d'identité. Je construis, crée un nouveau contexte pour ces sections, pour ces planchespeaux. Beaucoup plus qu'une récupération (dans l'ordre politico-économique), c'est, en fait, une réaffectation.

\section{Posture et position}

Cette réaffectation des éléments tirés du réel (toits, escaliers, murs, rampes, etc.) s'inscrit dans une réalité propre à l'oeuvre, dans un réalisme évident dont pourtant le sens et 


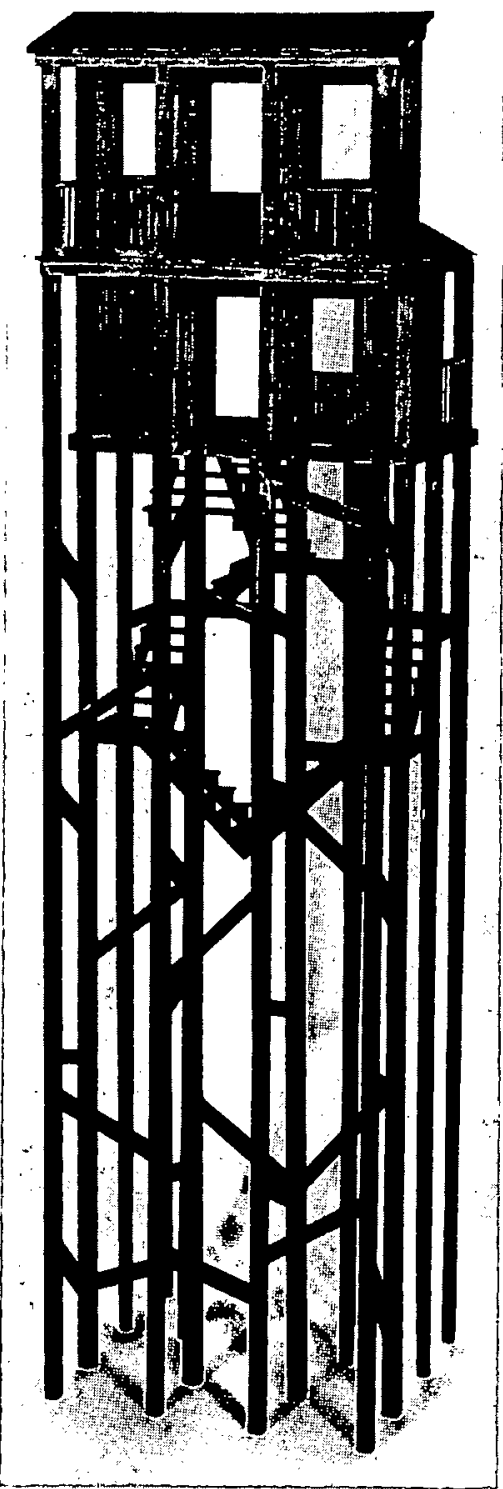

Marie-Chrystine Landry: Sans titre - bois, cuivre $1,02 \mathrm{~m} \times 27 \mathrm{~cm} \times 27 \mathrm{~cm}, 1989$ la fonction s'écroulent. La pièce (sculpture/ partie de sculpture) devient donc un espace intervallaire, un point d'aiguillage où le montré se retrouve à la limite d'une surcharge ou d'une absence, d'un fonctionnel ou d'une inefficacité. Se présentent autant de senssignes qui sont prêts à surgir ou à plonger dans la fiction, dans "l'enjouement * (la séduction, la passion du jeu, l'hédonisme des signes), dans le non-sens (et ce, parfois jusqu'à l'absurde), déjouant ainsi l'empreinte du réalisme. Ces signes-sens sont des provocateurs dé l'imagination, de l'affect. L'ensemble de ces signes circonscrit, marque, interpelle des espaces et des images dans une fantasmatique et un ordre mémorial propres à une collectivité. Mon travail réaffecte l'intime et le collectif pour tenter de saisir les rebords de chacun de ces territoires de moins en moins définis. Il se situe dans cette lisière intervallaire et jette un regard tant vers l'intérieur que vers l'extérieur, comme on peut le faire d'une galerie. Mon travail est parfois difficile à cerner ou à saisir et, comme pour tout intervalle, il faut s'y a(ban)donner ou prendre une chaise, s'asseoir et regarder.

Marie-Chrystine Landry 


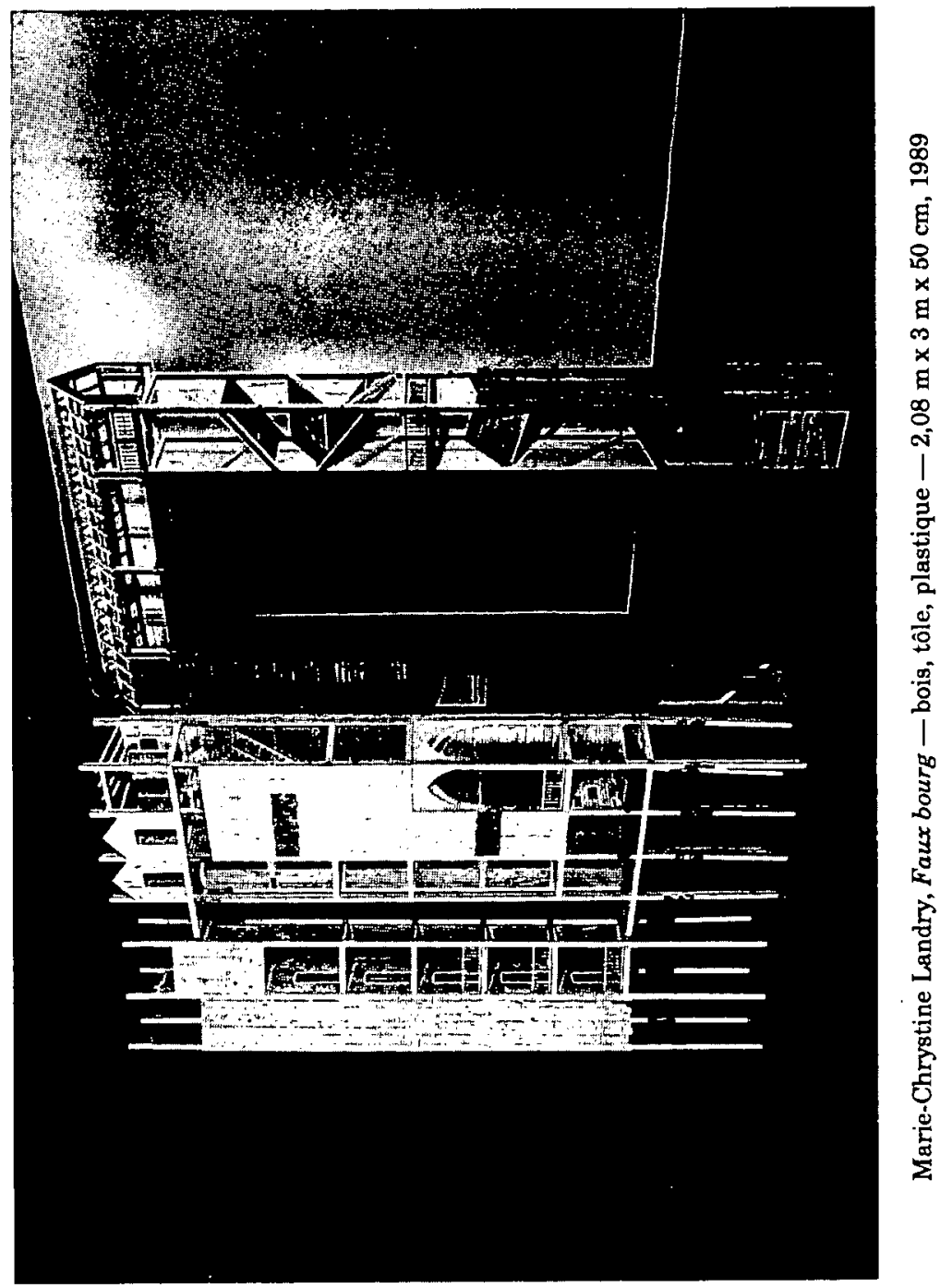


En présence d'une image qui rêve, il faut la prendre comme une invitation à continuer la rêverie qui l'a créée.

Gaston Bachelard, La poétique de l'espace

En ces temps de précipitation théorique (ou terminologique seulement?), au moment où la fine pointe écrivante se doit de dépasser le postmodernisme - n'a t-on pas déjà écrit en 1987 un Life after Postmodernism - pourquoi ne pas mettre les freins et ouvrir un vieux parapluie? Descendre des rayons les plus hauts de la bibliothèque quelque Bachelard jauni, un Emily $L$. d'avant le coma, quelques pétales de $L a$ pensée sauvage et autres néo-antiquités. Relire tranquillement, laisser travailler des sculptures de Marie-Chrystine Landry, faire un texte d'humeur. Oui, en revenir à une critique primesautière, à une dérive mi-formelle misociologique, les deux demies n'arrivant pas à joindre les deux bouts et à se constituer en traité ni même plus modestement en analyse d'œuvres. Penser et écrire dans les traces des Écrits timides sur le visible de Gilbert Lascault, petit recueil injustement relégué au tiroir 1979, où l'on pouvait lire entre autres considérations nonchalantes:

Pourtant la multiplicité diversifiée des tableaux, des sculptures peut nous aider à devenir moins raides, à nous éparpiller joyeusement. Parfois même, un seul tableau peut nous apprendre la dispersion, l'errance souple.

Se disperser, à partir de textes qui ne sont plus à la mode, mieux, qu'il n'est même plus de mise de contester. Reprendre en contrepoint les motifs du format réduit et du régionalisme, du petit espace et de l'excentrique.

\section{Des petites constructions}

Le brin de mousse peut bien être sapin, jamais sapin ne sera brin de mousse. L'imagination ne travaille pas dans les deux sens avec la même conviction.

Bachelard, La poétique de l'espace

Inutile de revenir longuement sur la question du fragment. D'autres en ont fait le tour, savamment ou joliment ou les deux à la fois (comme Anne Cauquelin dans un "court traité »). Et si l'on fait le tour d'un fragment, même en bonne 
86

compagnie, ne vient-on pas de lui donner une consistance exagérée, une importance hors proportion?

Oublions donc la question du fragment. Une sculpture, par exemple, ne saurait être qu'une partie d'un ensemble inexistant. *Un couteau sans lame auquel manque le manche *, disait saint Breton en reprenant l'aphorisme d'un autre qui ne vit désormais que par cette phrase répétée, un Lichtenberg qui n'a même pas droit au dictionnaire usuel.

Les sculptures/architectures seraient-elles alors des maquettes? Des fragments (zut, nous y revoilà) d'une maison ou d'un palais? Mais la maquette que l'on a émancipée, à laquelle on a donné un statut autonome, une vie sociale, est un projet, un non finito. La fin de la maquette coïncide exactement avec le début de. la construction. L'ingénieur matérialise l'architecture de papier et gomme le projet, transformant les personnages de carton indicateurs d'échelle en usagers. La maquette devient fétiche, parfois objet de musée, parfois photographie dans un album (selon la notoriété de son auteur). Le plus souvent, le petit projet retourne au placard pour en ressortir en pièces détachées au gré des autres projets. Objet d'uságe, la maquette s'aplatit.

Les sculptures/architectures - on dit aussi les architectures sculptures, l'indécision marquant aussi le lexique ne seront jamais habitables. L'utopie des sculpteurs des années 1960 (pensons ici à Roussil) quï rêvaient de sculptures habitables n'aura pas lieu, restera comme un souvenir piranésien, une utopie d'épóque. Cette poussée vers l'architecture sculptée serait d'ailleurs à revoir en tenant compte, par exemple, de l'indéfinition du métier de sculpteur d'alors qui aurait bien aimé se glisser dans la société en se rendant utile, qui aurait souhaité déloger l'ärchitecte et reprendre'les murs aux peintres.

Rien, ici, maintenant, d'ùn tel propos. Les sculptures de Landry, comme celles de Simonds; ne sont pas habitables. Que voulez-vous, ce ne sont paś des maquettes. La frustration n'est pas de mise. Nul ne résidera ici qui n'est pas d'abord un œil et tant pis pour les problèmes de plomberie.

Miniatures, alors? Encore une fois, le tremblé du lexique. Une sculpture serait en plus petit quelqué chose d'autre: un pont, un palais, une maison, une jetée ou un phare. Si j'agrandis la barque, si je la réalise au point de pouvoir ramer jusqu'à l'île, j'utilišé le motif comme maquette et la barque de 


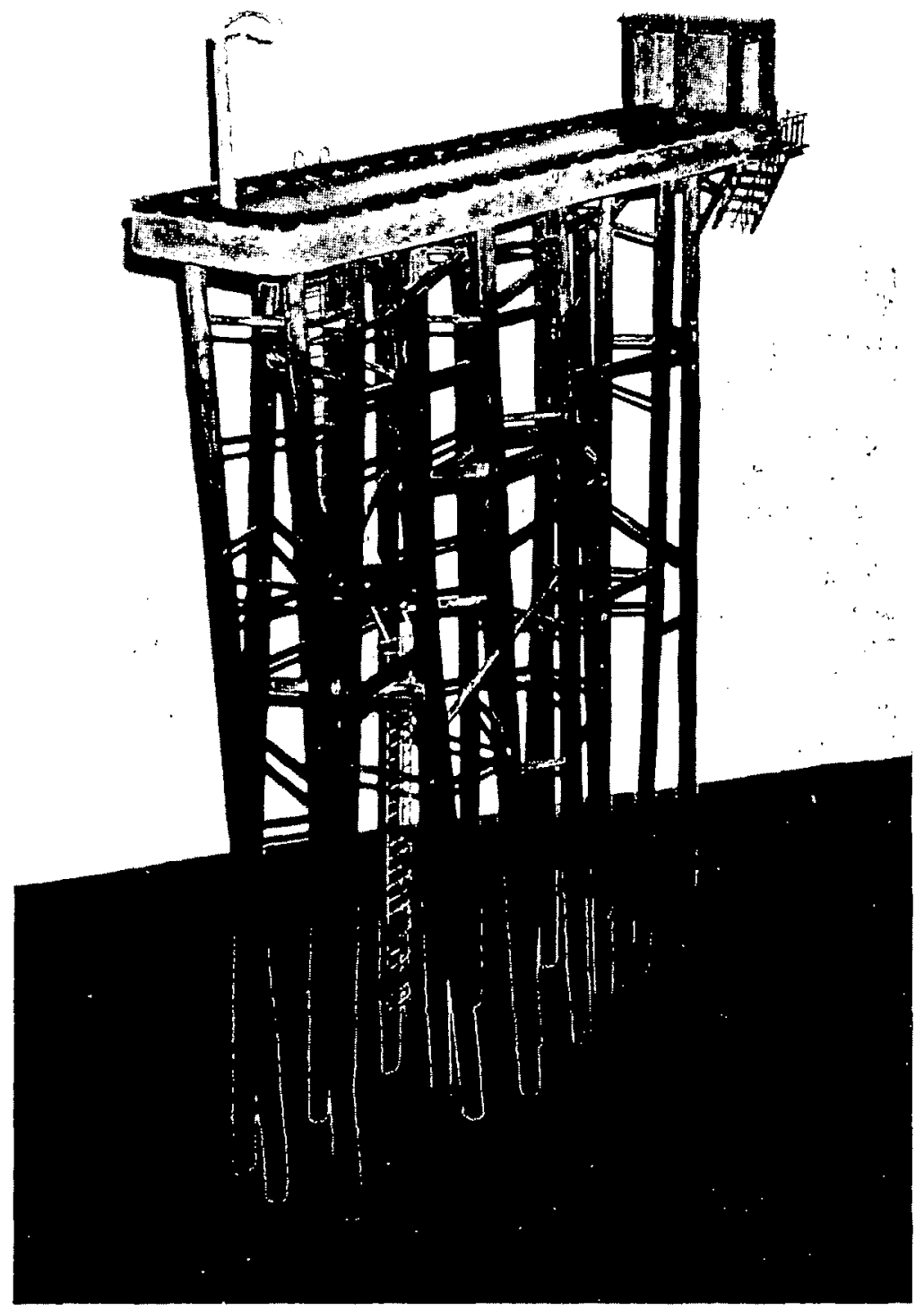

Marie-Chrystine Landry, Jetée - bois, tôle, ciment $1,11 \mathrm{~m} \times 28 \mathrm{~cm} \times 8 \mathrm{~cm}, 1988$ 
88

Landry prend l'eau. Qu'on n'y voit pas simple maladresse de ma part, impossibilité de construire une embarcation étanche. La barque, mode d'emploi? Très peu pour nous. L'œil citadin dérape, l'œil utilitaire coule. Qui me dira les dimensions de la jetée pour que je puisse la construire sur ma rivière? Si je dis miniature, je dois pouvoir mesurer un produit final: cette jetée serait trop longue pour ma rivière, trop courte pour votre golfe et envahissante dans une maison.

C'est à l'échelle de la sculpture qu'il faut travailler. La mode de l'installation (écrire le mode serait moins pernicieux, plus historien d'art que critique) permet aux artistes aujourd'hui - depuis un hier récent - d'envahir un espace. Le in situ a l'ego débordant, foisonnant. Autre manière de s'inscrire dans le social depuis que la sculpture habitable a perdu ses ténors: répandre ses métaphores personnelles (ses petites mythologies individuelles) mur à mur. Les * installateurs * seraient dans la position déjà signalée des rêveurs de sculptures habitables, souhaitant cette fois envahir l'intérieur de la maison, y déloger l'architecte, le peintre, aussi le sculpteur. L'ajout d'un vidéo mettrait la touche finale à l'éviction, en sortant TVA et Quatre-Saisons du salon. Dans un tel environnement - pour reprendre une expression post-Bachelard sans doute, mais tout aussi périmée - tout objet sculpté serait à lire comme une miniature. Mais qui pourrait donner la mesure d'une image qui rêve?

\section{La dispersion des centres}

Le soir qui vient toujours, lent, par couches successives, derrière les rangées de lampadaires, le long des routes du Havre sur l'autre rive. Et sur le fleuve qui devient noir. Un répit s'est produit dans les arrivages des clients. Ceux de la région sont déjà là. On attend encore des touristes toujours en retard sur les horaires français.

Marguerite Duras, Emily L

Quelques notes en marge, quelques mots échappés qui prendraient le fleuve à rebours, le lecteur à contre-pied. 
Trouver moyen de glisser en périphérie à partir d'un centre qui n'en est pas un, d'une ancienne métropole qui, parce qu'elle est une île, se croit transatlantique.

Comment entrer dans un monde riverain quand on vient d'une île, certes, mais tellement fabriquée, tellement trafiquée que les ponts ne sont qu'une autre façon de dire rues? Quand on vit sur une île avoisinante qui est bien un centre, mais commercial... Laval, pour la nommer, n'a pas de ccur: cette ville est une fabrication de promoteurs, un collage bureaucratique de petits villages devenues quartiers, certains morceaux ayant d'ailleurs perdu leur nom dans l'aventure. Qui se souvient de L'Abord-à-Plouffe qu'il fallait traverser pour se rendre à Pointe-Calumet en passant par SaintEustache, son terrain de golf, son église marquée par les canons du pouvoir lors du soulèvement des Patriotes? Peutêtre est-ce justement ce «qui se souvient » qui permet de passer à l'autre rive, une sorte de mémoire flottante qui ferait un pont de Laval à Rimouski.

Mémoire de l'une, ancrage du quotidien pour l'autre. "Ceux de la région sont déjà là. * Savoir aussi. Les sculpturespaysages, inscrites dans un lieu parce qu'elles y sont fabriquées, parce qu'elles tiennent compte d'un horizon différent et d'un fleuve noir (gris plomb, pense plutôt Michel Saulnier, riverain d'en bas), ces constructions donc sont aussi des sculptures. L'atelier est un centre informé: la maison qui en sortira vient tout aussi bien du versant roman d'Assise que d'une photographie glacée d'un catalogue d'exposition. Matériaux et couleurs du territoire immédiat certes ( il faut bien trafiquer quelque chose , disaient jadis Aragon/Ferré, "bricoler * écrivait Lévi-Strauss), mais construction syncrétique au sens ethnologique du terme, fusion d'éléments culturels différents. Une culture partagée repousse les frontières artificielles et, sans abolir le hasard (seul un coup de dé ferait l'affaire), permet le déplacement, l'errance, dans un même lieu ouvert aux interprétations qui n'en finissent pas.

"Quel serait alors un plaisir à ne pas achever *, dis-je en écho à Anne Cauquelin, riveraine de la rue Saint-Martin, qui sait bien, elle aussi, que le fleuve coule à l'envers et que le lieu n'est jamais un centre. 


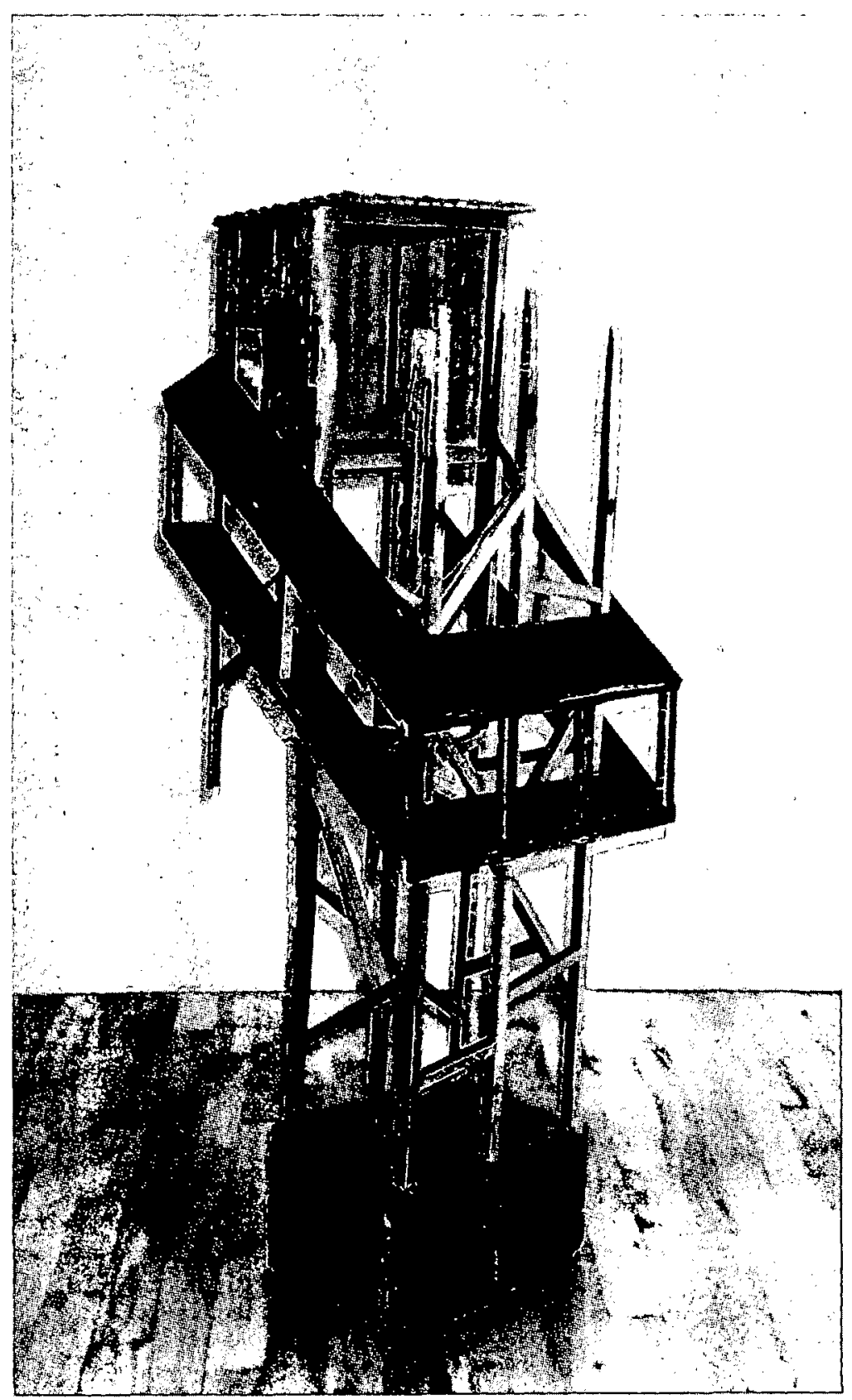

Marie-Chrystine Landry, Assise - bois, tôle, céramique $1,1 \mathrm{~m} \times 45 \mathrm{~cm} \times 35 \mathrm{~cm}, 1990$ 